Accretion Phenomena and Related Outflows, IAU Colloquium 163

ASP Conference Series, Vol. 121, 1997

D.T. Wickramasinghe, L. Ferrario, and G.V. Bicknell, eds.

\title{
Preface
}

\section{Dayal Wickramasinghe}

The Astrophysical Theory Centre, Australian National University, Canberra ACT 2601, Australia

The idea of holding a conference on accretion phenomena and related outflows in Australia was brought forward initially at the Padova conference on Cataclysmic Variables two years ago, and culminated in IAU Colloquium 163 held in Port Douglas, Australia between July 15-19 in 1996. The conference followed the general theme of the two previous very successful NATO Advanced Research Workshops - The Theory of Accretion Discs I and II held respectively in Paris in 1989, and in Germany in 1993, but was of wider in scope in that it embraced both theory and observations.

The conference brought together observers and theoreticians working on accretion phenomena and associated outflows in various astronomical systems, and covered many areas including, close interacting binaries, young stellar objects and active galactic nuclei. Over 200 participants attended from 17 countries. We were fortunate in attracting many of the leading researchers, and as a result were able to organise a relatively large number of review type presentations. The sessions were structured as far as possible to highlight common physics. This proved very successful, and held the attention of the participants for most of the conference, despite the obvious attractions of the Barrier Reef!.

On the theoretical side, significant progress had been made on several fronts. The problem of the angular momentum transport in accretion discs came in for much discussion. A new result was the demonstration that the well known hydrodynamic stability of Keplarian discs to linear perturbations appeared also to extend to the non- linear regime. The case for magnetic fields as the main agent responsible for turbulent viscosity in discs appeared to be stronger than ever before, and there was much discussion on the possibility of generating large scale magnetic fields by a disc dynamo.

There was some debate on the role played by advection-dominated accretion flow solutions as they relate to observed systems, and of the importance of accretion shocks. While it was clear that the classical geometrically thin Shakura- Sunyaev type accretion discs were an excellent approximation for explaining most disc-type phenomena in Cataclysmic Variables and X-ray binaries, it appeared that some low luminosity X-ray binaries and galactic X-ray sources were better explained by the new advection-dominated accretion flow solutions, or by accretion disc models involving shocks. Another interesting result was the suggestion that the warping of maser discs in AGN, and the precession of accretion discs in some X-ray binaries, may be related to radiation force induced torques.

The process of accretion on magnetised objects also came under close scrutiny, and there was much discussion on the properties of accretion discs, columns and curtains with interesting similarities drawn between different systems such as 
cataclysmic variables, X-ray binaries and T-tauri stars. Detailed observations of the disc-magnetic star interaction were provided by the new results from GRANATE of the spin-up and spin-down of neutron stars in X-ray binaries. Neutron stars appeared to show alternate spin-up and spin-down phases with characteristic torque profiles which are inconsistent with the standard model, and with ideas of spin equilibrium, indicating that these may need to be revisited.

Accretion onto blackholes was discussed under various contexts, ranging from x-ray and optical signatures of blackhole discs in AGN and binaries, to the presentation of new observations of the blackhole candidates recently discovered by the Compton Gamma Ray Observatory. The observations of the enigmatic black hole candidate GRO 1655-40 dominated this session, and although a model based on disc instability appeared to be the favoured explanation for the outburst phenomenon, it was unclear how the model could explain in detail the rich variety of phenomena, and time variability exhibited by this system.

Among the observations presented were recent Hubble Space Telescope images of young stellar objects and proto-stellar discs, tomography of accretion flows in cataclysmic variables and active galactic nuclei, and evidence of extremely luminous galactic mega-masers. In particular, observations were presented of YSOs in the Orion nebulae at resolutions of 10 s of astronomical units, revealing neutral discs and ionisation fronts. In addition, high resolution observations of supersonic jet outflows from YSOs have shown complex radiative shock structures presenting modelers with new challenges. The spectacular Hubble Space Telescope images were perhaps rivalled only by the quality and resolution of the numerical simulations presented at the conference. The progress in this area in the past few years appeared phenomenal, and there was hope that it would soon be possible to carry out 3-D MHD simulations of accretion and outflows with the detail required to explain observed morphologies, and inferred physical structures of discs and outflows in cataclysmic variables, young stellar objects, and active galactic nuclei.

A detailed summary of the scientific highlights of the conference can be found in the excellent review presented by Colin Norman.

A conference of the size of IAU Colloquium No 163 comprising over 200 participants required careful organisation, particularly since the conference was held some $2000 \mathrm{~km}$ from the host institute, the Astrophysical Theory Centre in Canberra. I would like to specially thank Lilia Ferrario, Chair of the LOC, who worked tirelessly for the better part of a year in meticulously planning the conference, leaving no detail to chance. Her judicious choice of conference venue was particularly appreciated by all who attended the conference. I am also grateful to Sarah Maddison for keeping a close eye on the organisation of the conference, for setting up and maintaining the Web page, and for her selfless efforts at Port Douglas in ensuring the smooth running of the conference. The support provided by the other members of the LOC are also acknowledged. Thanks are also due to $\mathrm{PhD}$ students Mike Burgess, Walid El Khouri and Roberto Soria for overseeing the question and answer sessions, Ralph Sutherland for general assistance with computing related matters, and Walter Stibbs for organising the poster sessions. 
I would like to extend my thanks to members of the Scientific Organising Committee for their advice in putting together the scientific program, which was particulalrly difficult given the diverse nature of the topics covered.

We are grateful to the various organisations which provided financial support, and made it possible for us to partially support students, young astronomers, invited speakers, and to defray some of the additional costs that were involved in holding the conference in Port Douglas. Significant grants were received from The Department of Industry, Science and Technology, The Australian National University in Canberra, and the US National Science Foundation. We also acknowledge support from the Research Centre for Theoretical Astrophysics, University of Sydney and the Anglo-Australian Observatory.

Finally, on behalf of the Editors, I would like to express our special thanks to Dr Stephen Meatheringham of Mount Stromlo and Siding Spring Observatories for his monumental efforts in collating the manuscripts and casting them into the format required by the publishers. 